\title{
Evaluation of third treatment week as temporal window for assessing responsiveness on repeated FDG-PET-CT scans in Non-Small Cell Lung Cancer patients
}

Citation for published version (APA):

Lazzeroni, M., Uhrdin, J., Carvalho, S., van Elmpt, W., Lambin, P., Dasu, A., Wersall, P., \& Toma-Dasu, I. (2018). Evaluation of third treatment week as temporal window for assessing responsiveness on repeated FDG-PET-CT scans in Non-Small Cell Lung Cancer patients. Physica Medica: European journal of medical physics, 46, 45-51. https://doi.org/10.1016/j.ejmp.2018.01.012

Document status and date:

Published: 01/02/2018

DOI:

10.1016/j.ejmp.2018.01.012

Document Version:

Publisher's PDF, also known as Version of record

\section{Document license:}

Taverne

\section{Please check the document version of this publication:}

- A submitted manuscript is the version of the article upon submission and before peer-review. There can be important differences between the submitted version and the official published version of record. People interested in the research are advised to contact the author for the final version of the publication, or visit the DOI to the publisher's website.

- The final author version and the galley proof are versions of the publication after peer review.

- The final published version features the final layout of the paper including the volume, issue and page numbers.

Link to publication

\footnotetext{
General rights rights.

- You may freely distribute the URL identifying the publication in the public portal. please follow below link for the End User Agreement:

www.umlib.nl/taverne-license

Take down policy

If you believe that this document breaches copyright please contact us at:

repository@maastrichtuniversity.nl

providing details and we will investigate your claim.
}

Copyright and moral rights for the publications made accessible in the public portal are retained by the authors and/or other copyright owners and it is a condition of accessing publications that users recognise and abide by the legal requirements associated with these

- Users may download and print one copy of any publication from the public portal for the purpose of private study or research.

- You may not further distribute the material or use it for any profit-making activity or commercial gain

If the publication is distributed under the terms of Article 25fa of the Dutch Copyright Act, indicated by the "Taverne" license above, 
Original paper

\title{
Evaluation of third treatment week as temporal window for assessing responsiveness on repeated FDG-PET-CT scans in Non-Small Cell Lung Cancer patients
}

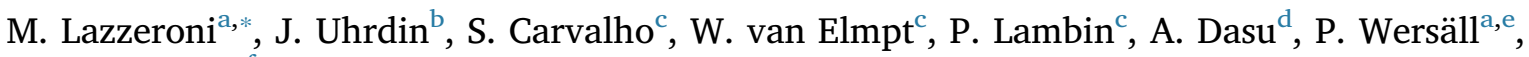 \\ I. Toma-Dasu ${ }^{\mathrm{a}, \mathrm{f}}$

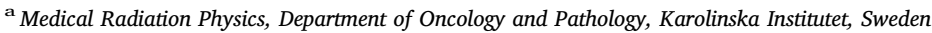 \\ ${ }^{\mathrm{b}}$ RaySearch Laboratories AB, Stockholm Sweden \\ ${ }^{c}$ Department of Radiation Oncology, GROW-School for Oncology and Developmental Biology, Maastricht University Medical Center, Maastricht, The Netherlands \\ d The Skandion Clinic, Uppsala, Sweden \\ e Karolinska University Hospital, Department of Medical Physics, Stockholm, Sweden \\ ${ }^{\mathrm{f}}$ Medical Radiation Physics, Department of Physics, Stockholm University, Sweden
}

\section{A R T I C L E I N F O}

\section{Keywords:}

FDG-PET-CT

NSCLC

Treatment adaptation

\begin{abstract}
A B S T R A C T
Purpose: Early assessment of tumour response to treatment with repeated FDG-PET-CT imaging has potential for treatment adaptation but it is unclear what the optimal time window for this evaluation is. Previous studies indicate that changes in $\mathrm{SUV}_{\text {mean }}$ and the effective radiosensitivity ( $\alpha_{\text {eff }}$, accounting for uptake variations and accumulated dose until the second FDG-PET-CT scan) are predictive of 2-year overall survival (OS) when imaging is performed before radiotherapy and during the second week. This study aims to investigate if multiple FDG-PET-derived quantities determined during the third treatment week have stronger predictive power.

Methods: Twenty-eight lung cancer patients were imaged with FDG-PET-CT before radiotherapy (PET1) and

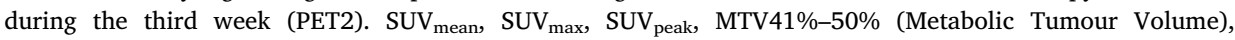
TLG41\%-50\% (Total Lesion Glycolysis) in PET1 and PET2 and their change (), as well as average $\alpha_{e f f}\left(\bar{\alpha}_{e f f}\right)$ and the negative fraction of $\alpha_{e f f}$ values $\left(f_{\alpha_{e f f}<0}\right)$ were determined. Correlations were sought between FDG-PET-derived quantities and OS with ROC analysis.

Results: Neither $\mathrm{SUV}_{\text {mean }}, \mathrm{SUV}_{\max }, \mathrm{SUV}_{\text {peak }}$ in PET1 and PET2 (AUC $=0.5-0.6$ ), nor their changes (AUC $=0.5-0.6)$ were significant for outcome prediction purposes. Lack of correlation with OS was also found for $\bar{\alpha}_{\text {eff }}(\mathrm{AUC}=0.5)$ and $f_{\alpha_{e f f}<0}(\mathrm{AUC}=0.5)$. Threshold-based quantities (MTV41\%-50\%, TLG41\%-50\%) and their changes had AUC $=0.5-0.7$.

P-values were in all cases $\gg 0.05$.

Conclusions: The poor OS predictive power of the quantities determined from repeated FDG-PET-CT images indicates that the third week of treatment might not be suitable for treatment response assessment. Comparatively, the second week during the treatment appears to be a better time window.
\end{abstract}

\section{Introduction}

The development and the clinical implementation of functional and molecular imaging during the last decades, together with sustained research work and innovations in imaging segmentation, registration and fusion, have imposed repeated imaging as one of the most promising tools for treatment response assessment [1]. The identification of predictive factors for tumour responsiveness at an early stage is fundamental for cancer treatment adaptation and individualisation. A patient-specific treatment adaptation, possibly through a dose escalation in the most radioresistant regions for the poor responders at risk of local recurrence, could improve treatment results $[2-5]$. ${ }^{18} \mathrm{~F}$-fluorodeoxy-D-glucose Positron Emission Tomography and Computed Tomography (FDG-PET-CT) have a great impact on cancer detection, staging, and treatment outcome assessment in a broad range of tumour types [6-8]. For lung cancer, several studies have already explored the possibility of identifying universal criteria for tumour responsiveness to chemoradiotherapy or radiotherapy alone from FDG-PET imaging

\footnotetext{
* Corresponding author at: Medical Radiation Physics, P9:02, Dept. of Oncology-Pathology, Karolinska Institutet, Box 260, S-171 76 Stockholm, Sweden.

E-mail address: Marta.Lazzeroni@ki.se (M. Lazzeroni).
} 
Table 1

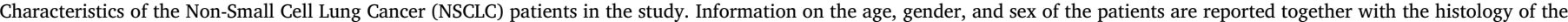

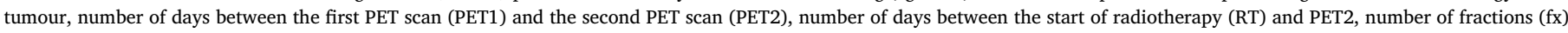
delivered by the time of PET2, dose delivered until PET2, and overall survival (OS) at 2 years (2Y) follow-up ( $1=$ survivor, $0=$ non-survivor).

\begin{tabular}{|c|c|c|c|c|c|c|c|c|c|}
\hline$\#$ & Age & Gender & Weight & Histology & Days between PET1 and PET2 & Days between start of RT and PET2 & Fx at PET2 & Dose at PET2 (Gy) & OS at $2 \mathrm{Y}$ \\
\hline 1 & 53 & $\mathrm{~F}$ & 64 & Adeno & 22 & 12 & 24 & 36 & 0 \\
\hline 2 & 48 & $\mathrm{~F}$ & 65 & Adeno & 24 & 13 & 26 & 39 & 1 \\
\hline 3 & 58 & $\mathrm{~F}$ & 48.5 & Adeno & 24 & 12 & 24 & 36 & 0 \\
\hline 4 & 59 & $\mathrm{~F}$ & 60 & Squam & 22 & 12 & 24 & 36 & 1 \\
\hline 5 & 63 & M & 84 & Squam & 21 & 12 & 24 & 36 & 0 \\
\hline 6 & 49 & M & 70 & Squam & 21 & 12 & 24 & 36 & 1 \\
\hline 7 & 67 & $\mathrm{~F}$ & 78 & Adeno & 31 & 14 & 28 & 42 & 0 \\
\hline 8 & 59 & $\mathrm{~F}$ & 54 & Other & 23 & 13 & 26 & 39 & 1 \\
\hline 9 & 66 & M & 66 & Squam & 21 & 12 & 24 & 36 & 1 \\
\hline 10 & 70 & M & 93 & Other & 23 & 13 & 26 & 39 & 0 \\
\hline 11 & 68 & M & 84 & Adeno & 25 & 12 & 24 & 36 & 0 \\
\hline 12 & 56 & $\mathrm{M}$ & 80 & Other & 27 & 15 & 30 & 45 & 1 \\
\hline 13 & 71 & M & 83 & Other & 21 & 12 & 24 & 36 & 1 \\
\hline 14 & 78 & $\mathrm{~F}$ & 63 & Other & 22 & 12 & 24 & 36 & 1 \\
\hline 15 & 60 & M & 79 & Squam & 21 & 12 & 24 & 36 & 1 \\
\hline 16 & 55 & $\mathrm{~F}$ & 91 & Adeno & 26 & 14 & 28 & 42 & 0 \\
\hline 17 & 66 & M & 78 & Squam & 20 & 12 & 24 & 36 & 1 \\
\hline 18 & 66 & $\mathrm{~F}$ & 50 & Other & 21 & 12 & 24 & 36 & 1 \\
\hline 19 & 64 & $\mathrm{M}$ & 103 & Squam & 22 & 12 & 24 & 36 & 0 \\
\hline 20 & 59 & $\mathrm{~F}$ & 61 & Other & 23 & 13 & 26 & 39 & 0 \\
\hline 21 & 67 & $\mathrm{~F}$ & 65 & Adeno & 25 & 12 & 24 & 36 & 1 \\
\hline 22 & 64 & M & 81 & Other & 21 & 12 & 24 & 36 & 1 \\
\hline 23 & 71 & $\mathrm{~F}$ & 64 & Other & 23 & 14 & 28 & 42 & 0 \\
\hline 24 & 58 & $\mathrm{~F}$ & 68 & Squam & 28 & 15 & 30 & 45 & 1 \\
\hline 25 & 38 & $\mathrm{~F}$ & 47 & Adeno & 21 & 9 & 18 & 27 & 1 \\
\hline 26 & 86 & M & 59 & Squam & 23 & 12 & 24 & 36 & 1 \\
\hline 27 & 52 & $\mathrm{M}$ & 78 & Adeno & 25 & 12 & 24 & 36 & 1 \\
\hline 28 & 69 & $\mathrm{~F}$ & 74 & Adeno & 22 & 12 & 24 & 36 & 0 \\
\hline
\end{tabular}

acquired after the completion of the treatment [9-12]. However, a limited number of investigations have so far accounted for changes in specific parameters at an early stage of chemoradiotherapy [13-17]. Recently, two independent studies on the same Non-Small Cell Lung Cancer (NSCLC) patient cohort have shown that different quantities can be calculated and were predictive of 2-year overall survival (OS) when imaging was performed before radiotherapy and during the second week $[18,19]$. Van Elmpt et al. [18] studied the change in the metabolic activity of the tumour based on the difference between the mean Standard Uptake Value (SUV), while Toma-Dasu et al. [19] adopted a volumetric approach and stratified the patients in good and poor responders based on calculations of an operational parameter, the effective radiosensitivity $\left(\alpha_{\text {eff }}\right)$.

In the assessment of the tumour response to treatment with repeated FDG-PET-CT imaging, the identification of the optimal window to performing the FDG-PET-CT scan during treatment is still unclear and few studies exist on the topic $[20,21]$. The time lapse between the first and the second FDG-PET-CT scan should be compatible with individualized treatment adaptation and should be carefully selected so that clonogenic cells are still detectable but the FDG-PET response is not affected to a large extent by the inflammatory reactions following chemoradiotherapy [22]. It could be hypothesised that the third week during the treatment might have a stronger predictive power on treatment outcome as compared to the second week since a larger portion of clonogenic cells has been depleted and therefore larger variations in the radiotracer uptake with respect to the initial FDG-PET image might be expected. At the same time, however, radiation induced inflammation might introduce a bias in the assessment of the uptake variation.

The present study therefore aims at assessing whether the third week of treatment could be a suitable time window for the assessment of tumour responsiveness as a predictor for the treatment outcome. In particular, parameters related to the distributions of $\alpha_{\text {eff }}$ values were for the first time tested for robustness of outcome prediction with respect to time window.

\section{Materials and methods}

\subsection{Treatment protocol and PET-CT acquisition}

Twenty-eight patients with advanced stage (stage III and IV) of NSCLC (10 adenocarcinoma cases, 9 squamous cell carcinoma cases, and 9 non-otherwise specified cases) were treated with concomitant chemoradiotherapy at the Maastricht University Medical Center (The Netherlands) between 2011 and 2012 and were analysed in this study. Patients enrolled in the clinical trial (NTC00522639 at clinicaltrials.gov) had no surgery and no previous cancer within five years prior to diagnosis. The administered chemotherapy agent was carboplatin-gemcitabine. The radiotherapy treatment consisted of two consecutive regimens of photon IMRT (Intensity Modulated RadioTherapy): a dose of $45 \mathrm{~Gy}$ was delivered in $1.5 \mathrm{~Gy}$ fractions twice per day followed by a dose-escalation up to $69 \mathrm{~Gy}$ in $2 \mathrm{~Gy}$ daily fractions.

The Xio/Focal System (Computerized Medical Systems, St. Louis, MO, USA) was used for radiotherapy planning. The software makes use of a convolution-superposition algorithm with inhomogeneity corrections. ICRU 50 [23] guidelines were adopted for treatment planning.

The primary Gross Tumour Volume $\left(\mathrm{GTV}_{\text {prim }}\right)$ was delineated on fused FDG-PET-CT images by experienced radiation oncologists.

Patients were imaged in radiotherapy position with FDG-PET-CT before the treatment and during the third week of radiotherapy. The mean and median time interval between the first FDG-PET-CT acquisition and the start of radiotherapy were equal to 11 and 10 days, respectively; while the mean and median time interval between the first and second FDG-PET-CT scan were both equal to 23 days.

The FDG-PET-CT acquisitions were performed with a Siemens Truepoint 40 camera (Siemens AG, Munich, Germany) in radiotherapy position. Patients fasted for at least $6 \mathrm{~h}$ before scanning and the NEDPAS [24] protocol was used with a FDG activity in MBq equal to 2.5 times the body weight in kg injected intravenously. FDG-PET image reconstruction was performed using an Ordered Subset Expectation 


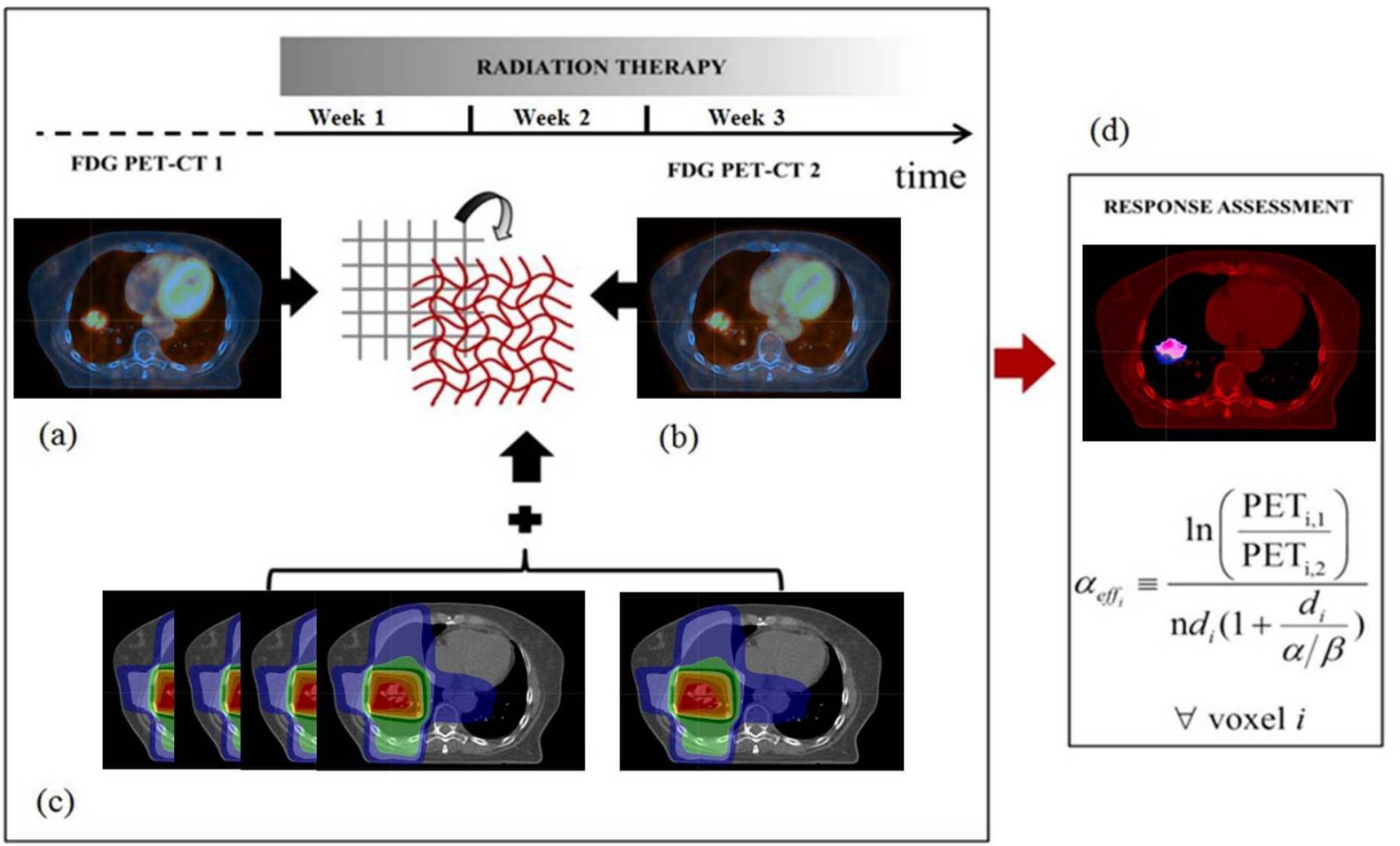

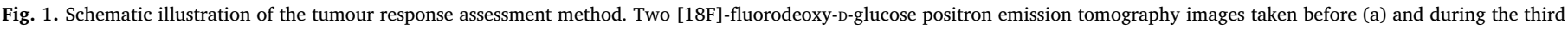

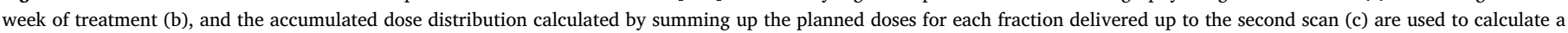
voxel-based effective radiosensitivity (d) and, thus, assess the responsiveness of the tumour to radiotherapy.

Maximization 2D four iterations eight subsets (OSEM2D 4i8s) algorithm. Corrections for random events and decay were applied, as well as scattering corrections using model-based methods. FDG-PET scans were also corrected for attenuation either using the mid-ventilation phase of the 4D-CT or a 3D-CT thorax. FDG-PET-CT scans were performed as static studies. No special breathing instructions were given to the patients, but normal breathing was recommended. CT and FDG-PET image voxel sizes were respectively equal to $(0.098 \times 0.098 \times 0.3) \mathrm{cm}^{3}$ and $(0.407 \times 0.407 \times 0.3) \mathrm{cm}^{3}$. Additional information on patient data, treatment protocol, and image data acquisition are described in Ref. [25].

Treatment outcome in terms of OS at 2 years was available for all the patients in the ensemble. Seventeen patients were alive at two years.

The patient characteristics most relevant for the presented analysis are listed in Table 1.

\subsection{Image registration and image analysis}

FDG-PET-CT scans acquired at the two successive time points were imported and co-registered in a research version of the treatment planning system RayStation version 4.6.100.4 (RaySearch Laboratories AB). Specifically, the two CT images, inherently registered to the FDGPET scans, were co-registered to achieve a voxel-to-voxel correlation between the two corresponding FDG-PET images. The registration consisted of a rigid registration based on bony anatomy followed by a hybrid deformable registration. The latter combines image information with anatomical information as provided by contoured image sets and uses as controlling region the external patient contour aiming at anatomical structure integrity [26].

Co-registered FDG-PET images were exported from the RayStation and analysed with an in-house MatLab code (The MathWorks Inc., Natick, MA, version R2011b) able to handle FDG-PET-CT images, dose distribution and delineated structures outside RayStation as well as to calculate the quantities of interest to perform the rest of the analysis.

\subsection{Calculation of FDG-PET-related quantities of interest}

To assess tumour early responsiveness to the treatment, a systematic voxel-based analysis using the co-registered FDG-PET images taken before radiotherapy and during the third week in the primary gross tumour volume $\left(\mathrm{GTV}_{\text {prim }}\right)$ was performed. The method, developed by Toma-Dasu et al. [19], takes into account the local uptake changes in the target volume of the paired FDG-PET images and the planned accumulated dose distribution at the second FDG-PET acquisition. The algorithm assumes that the uptake variation at voxel level in the images is proportional to the variation of functional clonogenic cell density and it is based on the Linear Quadratic (LQ) [27] formalism for cell killing. The same radiosensitivity to fractionation (i.e. $\alpha / \beta=10 \mathrm{~Gy}$ for the tumour) is assumed for all the patients and an operational parameter, the effective radiosensitivity $\alpha_{e f f}$, is calculated:

$$
\alpha_{e f f, i}=\frac{\ln \left(\frac{P E T_{i, 1}}{P E T_{i, 2}}\right)}{n d_{i}\left[\frac{1+d_{i}}{(\alpha / \beta)}\right]},
$$

where $P E T_{i, 1}$ and $P E T_{i, 2}$ are the SUV values in the FDG-PET images for the voxel of interest $i$ acquired before and during radiotherapy, respectively; $d_{i}$ and $n$ are the planned dose per fraction and the number of fractions delivered until the second FDG-PET image, respectively.

A positive value of the effective radiosensitivity parameter indicates a decrease in the tracer uptake of the FDG-PET scan acquired during the treatment with respect to the initial FDG-PET scan for a certain voxel under consideration and a negative value indicates an increase, respectively.

The distribution of the effective radiosensitivity, $\alpha_{\text {eff }}$, in each voxel in a volume of interest is therefore obtained. The average value of the effective radiosensitivity, $\bar{\alpha}_{e f f}$, and the fraction of negative values of $\alpha_{e f f}$, $f_{\alpha_{e f f}<0}$, are also calculated. The volume of interest in this study in which the distribution of the effective radiosensitivity was assessed was $\mathrm{GTV}_{\text {prim. }}$. 
A schematic illustration of the method for assessing the early tumour responsiveness to treatment is presented in Fig. 1.

Additionally, other FDG-PET-derived quantities were also scored in the first and second FDG-PET scan. The mean and the maximum SUV values $\left(\mathrm{SUV}_{\text {mean }}, \mathrm{SUV}_{\text {max }}\right)$ in the first and second FDG-PET scan were scored in the volume corresponding to $\mathrm{GTV}_{\text {prim. }}$. Following the European Association of Nuclear Medicine (EANM) guidelines for tumour imaging with FDG-PET-CT (version 2.0, [28]), SUV peak $_{\text {was }}$ scored in a $1.2 \mathrm{~cm}$ diameter spherical volume of interest (VOI) positioned such that the average value across all positions within the lesion is maximised. Threshold-based quantities such as the Metabolic Tumour Volume (MTV) and the corresponding Total Lesion Glycolysis (TLG) were also considered in the study. The MTV41\% and MTV50\% corresponded, respectively, to a 3D isocontour at $41 \%$ of the maximum pixel value and to a 3D isocontour at $50 \%$ of the maximum pixel value [28]. The TLG values (TLG41\% and TLG50\%, respectively) were obtained as the product of the mean SUV value in the MTV VOI and the MTV volume (MTV41\% and MTV50\%, respectively) [28].

The percent change in the maximum SUV $\left(\Delta \mathrm{SUV}_{\mathrm{max}}\right)$ within $\mathrm{GTV}_{\text {prim }}$ between the FDG-PET scans before the treatment $\left(\mathrm{SUV}_{\max , 1}\right)$ and during the third week $\left(\mathrm{SUV}_{\max , 2}\right)$ was calculated as $\Delta \mathrm{SUV}_{\max }=100$ $\left(S U V_{\text {max }, 1}-S U V_{\max , 2}\right) / S U V_{\text {max }, 1}$. Similarly, the percent changes in the other FDG-PET-derived quantities mentioned above $\left(\Delta \mathrm{SUV}_{\text {mean }}\right.$, $\Delta$ SUV $_{\text {peak }}, \Delta \mathrm{MTV} 41 \%, \Delta \mathrm{MTV} 50 \%, \Delta \mathrm{TLG} 1 \%$, and $\Delta \mathrm{TLG50} \%$ ) were calculated.

\subsection{Statistical analysis}

The statistical analysis was performed by using the Receiver Operating Characteristic (ROC) method to seek for correlations between the treatment outcome of NSCLC patients, expressed as 2-year OS, and the quantities of interest mentioned above $\left(\bar{\alpha}_{\text {eff }}, f_{\alpha_{\text {eff }}<0}\right.$, $\mathrm{SUV}_{\text {max }}, \mathrm{SUV}_{\text {mean }}, \mathrm{SUV}_{\text {peak }}, \mathrm{MTV}_{4} \%$, MTV50\%, TLG41\% and TLG50\% in PET1 and PET2, the percentage change of SUV ${ }_{\text {mean }}, \mathrm{SUV}_{\text {max }}, \mathrm{SUV}_{\text {peak, }}$ MTV41\%, MTV50\%, TLG 41\% and TLG50\%). The Area Under the ROC curve (AUC) and corresponding p-values were scored. A p-value below 0.05 was considered statistically significant.

The commercial software, MedCalc, version 13.1.0.0 (MedCalc Software, Ostend, Belgium) was used for the calculations.

\section{Results}

Fig. 2 shows the distribution of the percent change in the FDG-PETderived quantities analysed in the study $\left(\mathrm{SUV}_{\text {mean }}, \mathrm{SUV}_{\text {max }}, \mathrm{SUV}_{\text {peak }}\right.$, MTV41\%, MTV50\%, TLG 41\% and TLG50\%), together with the distributions of $\bar{\alpha}_{e f f}$ and $f_{\alpha_{e f f}<0}$ for the NSCLC cohort under investigation. Patients were divided based on the 2-year OS as survivors and nonsurvivors. As seen, the distribution of the data is similar for survivors (black) and non-survivors (red) in all cases, as evidenced also by the similar median and mean values of the distributions (empty square and horizontal line, respectively, in the box plot).

Fig. 3 shows the ROC curves investigating the correlation between all the FDG-PET-derived quantities under analysis in this study and the treatment outcome. As reasonably expected from the results shown in Fig. 2, the ROC analysis indicates that none of the considered quantities has a statistically significant discriminant power. AUC values for $\bar{\alpha}_{\text {eff }}$ and $f_{\alpha_{\text {eff }}<0}$ were, respectively, AUC $=0.5$ with $\mathrm{p}=0.7$ and $\mathrm{AUC}=0.5$ with $\mathrm{p}=0.8$. The change between the FDG-PET uptake in PET1 and PET2 did not correlate with the outcome either and resulted in an $\mathrm{AUC}=0.6$ with $\mathrm{p}=0.3$ for $\Delta \mathrm{SUV}_{\max }, \mathrm{AUC}=0.5$ with $\mathrm{p}=0.7$ for $\Delta \mathrm{SUV}_{\text {mean }}$, and $\mathrm{AUC}=0.6$ with $\mathrm{p}=0.4$ for $\Delta \mathrm{SUV}_{\text {peak. }}$. Similar results are also found for the $S_{U V}$ max,$S_{\text {mean }}$, and $S_{U V}$ peak values in PET1 or PET2 having all AUC values in the range $0.5-0.6$ with p-values between 0.5 and 0.9 .

Threshold-based methods (MTV41\%, MTV50\%, TLG41\%, and
TLG50\%) calculated in PET1 and PET2 did not result in better predictive power either with an AUC value range equal to $0.5-0.7$ and pvalues in the range $0.2-0.7$. Percentage difference of the mentioned threshold-based quantities, i.e. $\Delta \mathrm{MTV} 41 \%, \Delta \mathrm{MTV} 50 \%, \Delta \mathrm{TLG} 41 \%$, and $\Delta$ TLG50\%, had AUC values respectively equal to $0.7(\mathrm{p}=0.1), 0.6$ $(\mathrm{p}=0.2), 0.6(\mathrm{p}=0.5)$, and $0.6(\mathrm{p}=0.3)$.

\section{Discussion}

One of the most quoted reasons of concern when assessing the response to treatment of patients receiving chemoradiotherapy based on repeated FDG-PET-CT imaging at different time points during the course of the treatment is related to the possibility of quantifying the metrics used in the comparison in an artefact-free manner [20,22]. Thus, the potentially radiation-induced image misrepresentations due to, for example, tissue inflammations and the way they might affect the results should be taken into account. The feasibility of designing metrics based on significant changes in the SUV values at different time points during the course of chemoradiotherapy for NSCLC patients has been shown in the study by Edet-Sanson et al. [20]. The authors aimed at verifying whether significant changes in the uptake of FDG or in the volumes defined based on the tracer uptake could be determined at different points during the course of a conventionally fractionated radiotherapy treatment, early enough to allow treatment adaptation. They performed 5-6 FDG-PET-CT scans, one every 7 fractions of $2 \mathrm{~Gy}$, during the course of the treatment for 10 NSCLC patients. Their results lead to the conclusion that although the normalised $\mathrm{SUV}_{\max }$ determined at different time points within the treatment could be determined in a radiation-induced misrepresentation-free manner, other parameters, depending on the size of the volume delineated using FDG-PET-based thresholds, were not reliable after about one to two weeks. However, this study did not include in the analysis the correlation between the tracer uptake and the treatment outcome. A prospective subsequent study by the same group, investigated the potential of using FDG-PETbased metrics and their changes during chemoradiotherapy as predictive factors for the treatment outcome at 1 year, expressed as OS or tumour progression [21]. The first FDG-PET image was acquired right before the start of the radiotherapy and the second image five weeks within the treatment after the delivery of $40-45 \mathrm{~Gy}$. They concluded that the only parameter that was actually able to predict the outcome at 1 year was $S_{U V} V_{\text {max }}$ in the second FDG-PET image taken at 5 weeks after the start of the treatment. Furthermore, Vera et al. [21] showed that the fixed threshold methods, based on FGD uptake, such as $40 \% \mathrm{SUV}_{\max }$, tend to delineate larger volumes even when the lesions were obviously decreasing at visual examination. It seems plausible to hypothesize that radiation-induced inflammation or similar processes is the reason for this increase in size. To some extent, the present and the above described studies point to the similar conclusion that beyond 1-2 weeks within the treatment the delineation of volumes based on FDG-PET becomes problematic. Consequently, the derivation of related parameters, such as SUV mean and its changes, becomes unreliable and leads to poor correlation with the treatment outcome with the exception just mentioned (i.e. $\mathrm{SUV}_{\max }$ at 5 weeks during the treatment). Nonetheless, a direct comparison between the findings of our study and the one by Vera et al. [21] is not entirely relevant because of the non-identical manner in which the analysed parameters were derived. The predictive value of repeated FDG-PET-CT for the treatment outcome for NSCLC patients was also investigated in a recent study by Mattoli et al. [29] concluding that early metabolic response was predictive for local control, progression free survival and overall survival after low-dose fractionated radiotherapy with concurrent chemotherapy. However, a direct comparison of the findings of the study by Mattoli et al. and the present one is not possible due to the different treatment regimens employed for the patient cohorts in the two studies.

The analysis of the tumour responsiveness performed in this study based on the effective radiation sensitivity, $\alpha_{\text {eff }}$, did not lead to better 

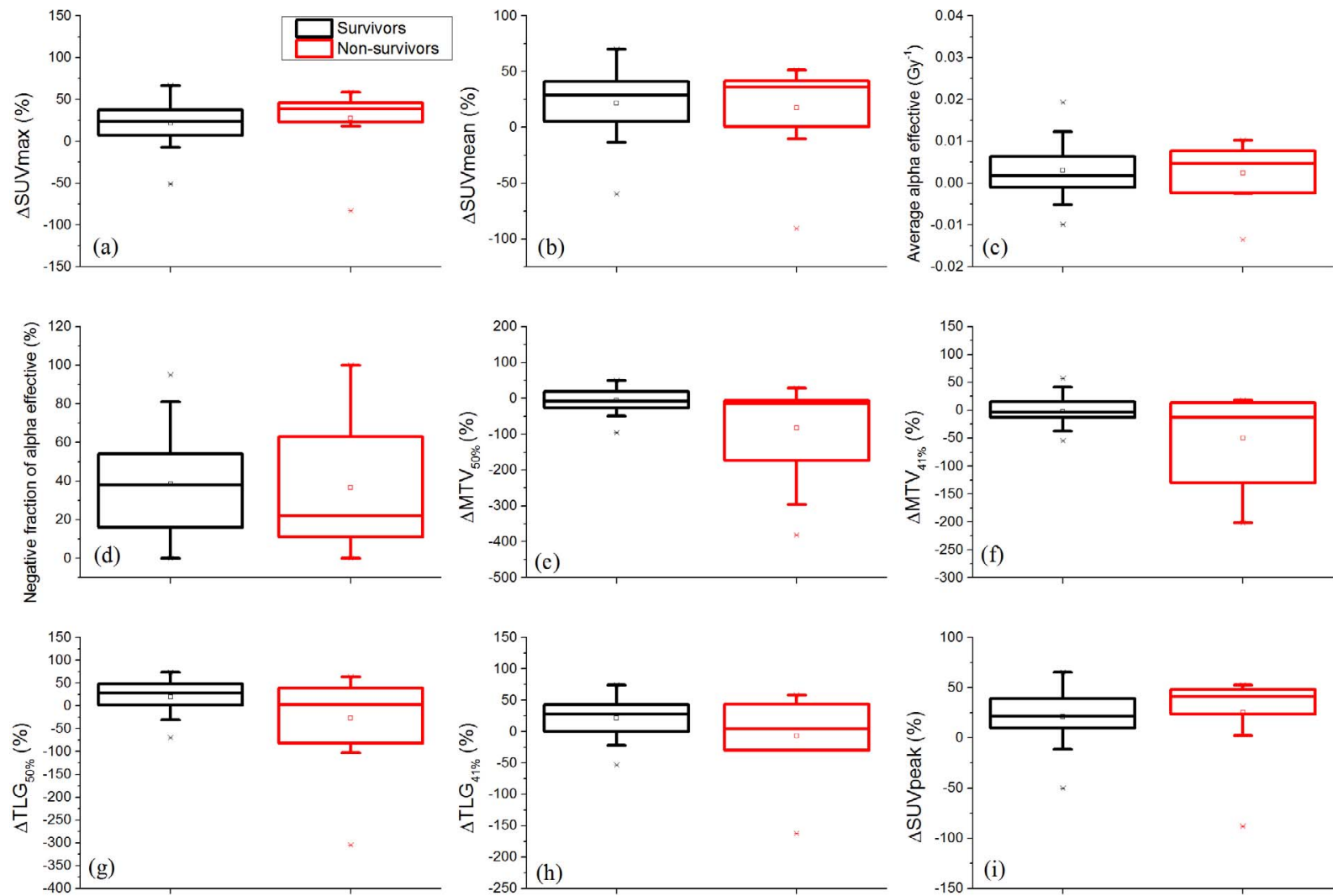

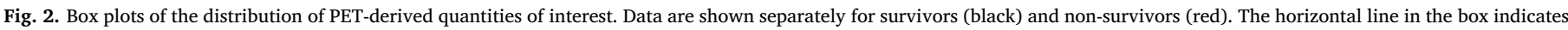

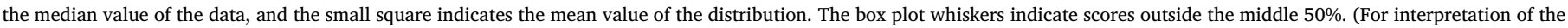
references to colour in this figure legend, the reader is referred to the web version of this article.)

predictions of the treatment outcome either, although it is not depending on a volume delineated based on FDG-PET uptake but strictly determined within the $\mathrm{GTV}_{\text {prim }}$. Beyond the possible radiation-induced inflammation problem, one possible explanation for this result is the actual definition of the effective radiation sensitivity. In fact, $\alpha_{e f f}$ implicitly assumes that the decrease in the FDG tracer uptake implies effective cell killing and an increase in the FDG uptake indicates that cell proliferation has a larger effect than treatment induced cell depletion. However, one has to keep in mind that the signal in the image is not given only by the surviving cells that metabolise the tracer but also by the damaged cells which are doomed to die but are still viable at the time of the scan and still capable of metabolising the tracer [22]. The proportion of the surviving clonogens in the entire cell population in the target, including the damaged clonogens doomed to die, varies at different moments during the treatment and therefore the discriminatory power of a metric for tumour response based on this is also expected not to be the same at different time points during the treatment.

The approach based on repeated examinations performed for the same patient in order to determine the effective radiation sensitivity, $\alpha_{\text {eff }}$, provides however one strong advantage. By using each patient as its own reference, no specific assumptions regarding the radiosensitivity of the patient would be required. In addition, according to the assumed approach for calculation of the $\alpha_{e f f}$ distributions, each voxel is its own reference and this avoids adding to the analysis unnecessary uncertainties associated with making specific assumptions regarding the initial cell density or proliferation rates in the voxels or possible causes for persistent uptake in the voxels. Uncertainties deriving from, for example, the limited resolution of the PET images and partial volume effects are also expected to be less pronounced. No specific strategy at the moment of the image acquisition was adopted to reduce the motion due breathing of the patient (except for normal breathing recommendation) under the assumption that the possible blurring of the PET images would be overpassed by uncertainties related to the co-registration of images (FDG-PET-CT1 and FDG-PETCT2).

The algorithm connected with the hybrid deformable registration was extensively tested and validated and found to perform well in comparisons to other algorithms, as discussed in Ref. [26]. The method for calculation of $\alpha_{\text {eff }}$ distributions was also tested for robustness by intentionally perturbing the registration between the FDG-PET images in Ref. [19]. Rigid $5 \mathrm{~mm}$ translations in anterior-posterior, left-right, and cranial-caudal directions were respectively introduced in the registration matrix. The classification of patients in responders and nonresponders to treatment resulted to be robust against the performed perturbation [19].

A limitation of the present study is represented by the relatively low number of patients $(\mathrm{N}=28)$. Unfortunately, this is a common problem in this kind of investigations (e.g. $\mathrm{N}=34$ in Van Elmpt et al. [18], $\mathrm{N}=26$ in Toma-Dasu et al. [19], and $\mathrm{N}=10$ in Edet-Sanson et al. [20]). Needless to say that the study would benefit from being repeated on a larger number of patients. In event of a positive correlation between the tumour responsiveness metrics and the treatment outcome, a cross-validation would have to be performed as in the previous study by Toma-Dasu et al. [19] on a similar number of patients imaged at 2 weeks from the start of the treatment but here it was not the case. So, one could only conclude from the current analysis that FDG-PET imaging at three weeks into treatment does not give any prognostic/predictive value for this dataset.

In summary, the lack of correlation of SUV $\mathrm{max}_{\text {max }}, \mathrm{SUV}_{\text {mean }}, \mathrm{SUV}_{\text {peak }}$, MTV41\%, MTV50\%, TLG41\%, and TLG50\% and their percent changes between the FDG-PET scans before the treatment and during the third week with OS indicates that the third week of treatment might not be suitable for assessing the responsiveness to treatment and subsequent 

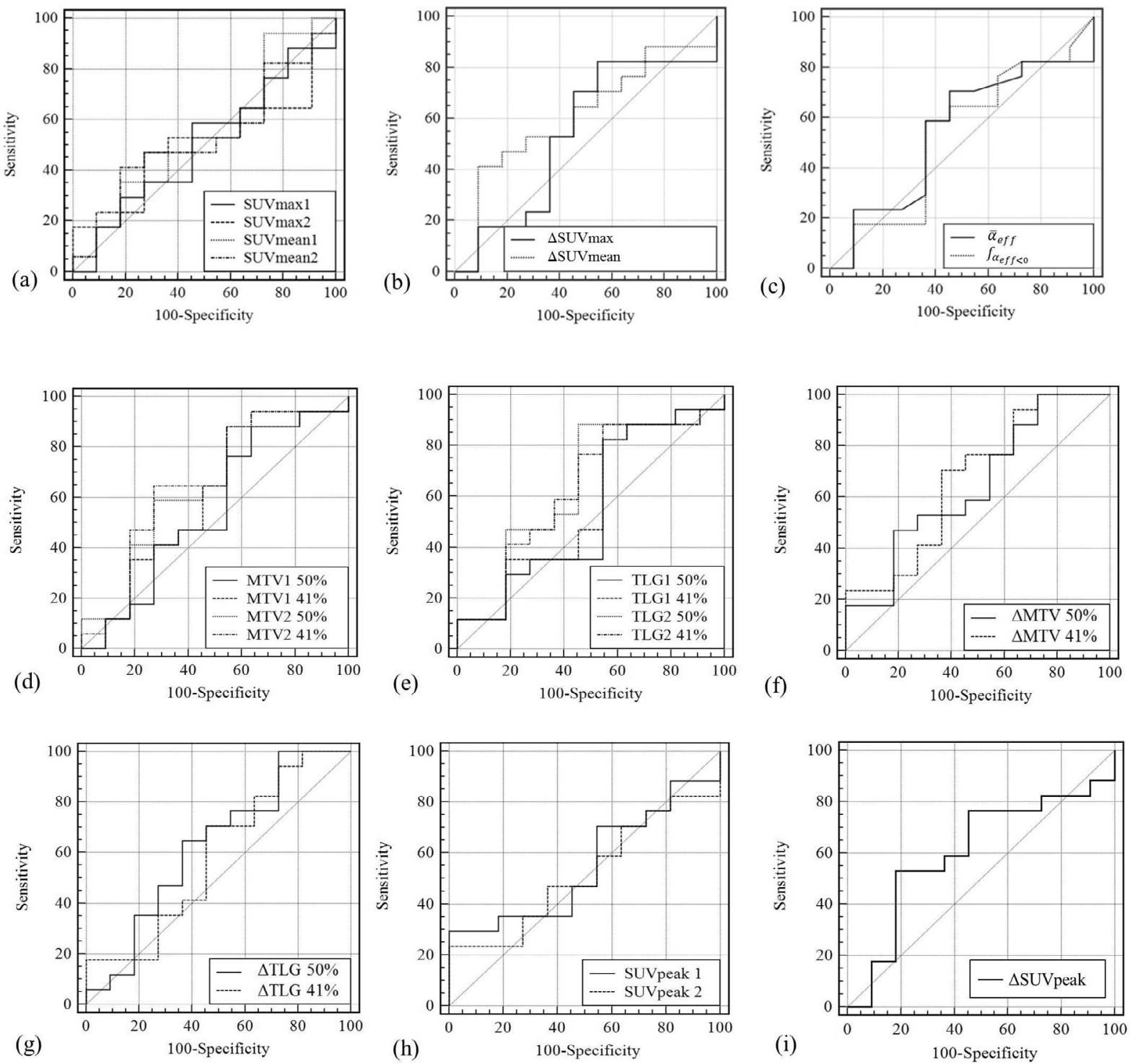

Fig. 3. ROC analysis. The correlation between PET-derived quantities of interest and the overall survival at 2 years is investigated.

treatment adaptation. In addition, the lack of correlation between the parameters derived from the distribution of the calculated effective radiosensitivity at three weeks from the start of the treatment with the treatment outcome points towards the same conclusion. Comparing with previous similar studies, the second week during the treatment appears to be a better time window for response assessment.

\section{Conflict of interest declaration}

Johan Uhrdin is employed by RaySearch Laboratories AB. The authors report no other conflicts of interest.

\section{Acknowledgments}

This research was supported by EU FP7 funding (ARTFORCE). Additional financial support from the Cancer Research Funds of Radiumhemmet is also acknowledged.

\section{References}

[1] Toma-Dasu I, Dasu A. Towards multidimensional radiotherapy: key challenges for treatment individualisation. Comput Math Methods Med 2015;2015:1-8.

[2] Feng M, Kong FM, Gross M, Fernando S, Hayman JA, Ten Haken RK. Using fluorodeoxyglucose positron emission tomography to assess tumour volume during radiotherapy for non-small-cell lung cancer and its potential impact on adaptive dose escalation and normal tissue sparing. Int J Radiat Oncol Biol Phys 2009;73:1228-34.

[3] Gillham C, Zips D, Pönisch F, Evers C, Enghardt W, Abolmaali N, et al. Additional PET/CT in week 5-6 of radiotherapy for patients with stage III non-small cell lung cancer as a means of dose escalation planning? Radiother Oncol 2008;88:335-41.

[4] van Elmpt W, De Ruysscher D, van der Salm A, Lakeman A, van der Stoep J, Emans $\mathrm{D}$, et al. The PET-boost randomised phase II dose-escalation trial in non-small cell lung cancer. Radiother Oncol 2012;104:67-71.

[5] Moller DS, Khalil AA, Knap MM, Muren LP, Hoffmann L. A planning study of radiotherapy dose escalation of PET-active tumour volumes in non-small cell lung cancer patients. Acta Oncol 2011;50:883-8.

[6] Bomanji JB, Costa DC, Ell PJ. Clinical role of positron emission tomography in oncology. Lancet Oncol 2001;2:157-64.

[7] Bussink J, van Herpen CM, Kaanders JH, Oyen WJ. PET-CT for response assessment and treatment adaptation in head and neck cancer. Lancet Oncol 2010:11:661-9.

[8] Van de Wiele C, Lahorte C, Oyen W, Boerman O, Goethals I, Slegers G, et al. Nuclear medicine imaging to predict response to radiotherapy: a review. Int J Radiat Oncol Biol Phys 2003;55:5-15.

[9] Abramyuk A, Tokalov S, Zophel K, Koch A, Szluha Lazanyi K, Gillham C, et al. Is pre-therapeutical FDG-PET/CT capable to detect high risk tumour subvolumes responsible for local failure in non-small cell lung cancer? Radiother Oncol 2009;91:399-404.

[10] Aerts HJ, Bosmans G, van Baardwijk AA, Dekker AL, Oellers MC, Lambin P, et al. Stability of $18 \mathrm{~F}$-deoxyglucose uptake locations within tumour during radiotherapy for NSCLC: a prospective study. Int J Radiat Oncol Biol Phys 2008;71:1402-7.

[11] Mac Manus MP, Hicks RJ, Matthews JP, McKenzie A, Rischin D, Salminen EK, et al Positron emission tomography is superior to CT scanning for response assessment 
after radical radiotherapy/chemoradiotherapy in patients with non- small-cell lung cancer. J Clin Oncol 2003;21:1285-92.

[12] Aerts HJWL, van Baardwijk AAW, Petit SF, Offermann C, Loon Jv, Houben R, et al. Identification of residual metabolic-active areas within individual NSCLC tumours using a pre-radiotherapy 18Fluorodeoxyglucose-PET-CT scan. Radiother Oncol 2009;91:386-92.

[13] Huang W, Zhou T, Ma L, Sun H, Gong H, Wang J, et al. Standard uptake value and metabolic tumor volume of 18F-FDG PET/CT predict short-term outcome early in the course of chemoradiotherapy in advanced non-small cell lung cancer. Eur J Nucl Med Mol Imaging 2011;38:1628.

[14] Kong F-MS, Frey KA, Quint LE, Haken RKT, Hayman JA, Kessler M, et al. A pilot study of $[18 \mathrm{~F}]$ fluorodeoxyglucose positron emission tomography scans during and after radiation-based therapy in patients with non-small-cell lung cancer. J Clin Oncol 2007;25:3116-23.

[15] Massaccesi M, Calcagni ML, Spitilli MG, Cocciolillo F, Pelligrò F, Bonomo L, et al. 18 F-FDG PET-CT during chemo-radiotherapy in patients with non-small cell lung cancer: the early metabolic response correlates with the delivered radiation dose. Radiat Oncol 2012;7:106.

[16] Usmanij EA, Geus-Oei L-Fd, Troost EGC, Peters-Bax L, van der Heijden EHFM, Kaanders JHAM, et al. 18F-FDG PET early response evaluation of locally advanced non-small cell lung cancer treated with concomitant chemoradiotherapy. J Nucl Med 2013;54:1528-34.

[17] van Baardwijk A, Bosmans G, Dekker A, van Kroonenburgh M, Boersma L, Wanders $\mathrm{S}$, et al. Time trends in the maximal uptake of FDG on PET scan during thoracic radiotherapy. A prospective study in locally advanced non-small cell lung cancer (NSCLC) patients. Radiother Oncol 2007;82:145-52.

[18] van Elmpt W, Öllers M, Dingemans A-MC, Lambin P, De Ruysscher D. Response assessment using 18F-FDG PET early in the course of radiotherapy correlates with survival in advanced-stage non-small cell lung cancer. J Nucl Med 2012;53:1514-20.

[19] Toma-Dasu I, Uhrdin J, Lazzeroni M, Carvahlo S, van Elmpt W, Lambin P, et al. Evaluating tumor response of non-small cell lung cancer patients with (18)F-fludeoxyglucose positron emission tomography: potential for treatment individualization. Int J Radiat Oncol Biol Phys 2015;91:376-84.

[20] Edet-Sanson A, Dubray B, Doyeux K, Back A, Hapdey S, Modzelewski R, et al. Serial assessment of FDG-PET FDG uptake and functional volume during radiotherapy (RT) in patients with non-small cell lung cancer (NSCLC). Radiother Oncol 2012;102:251-7.

[21] Vera P, Mezzani-Saillard S, Edet-Sanson A, Menard JF, Modzelewski R, Thureau S, et al. FDG PET during radiochemotherapy is predictive of outcome at 1 year in nonsmall-cell lung cancer patients: a prospective multicentre study (RTEP2). Eur J Nucl Med Mol Imaging 2014;41:1057-65.

[22] Brahme A. Biologically optimized 3-dimensional in vivo predictive assay-based radiation therapy using positron emission tomography-computerized tomography imaging. Acta Oncol 2003;42:123-36.

[23] ICRU Report 50: Prescribing, Recording and Reporting Photon Beam Therapy. Bethesda, MD: International Commission on Radiation Units and Measurements; 1993.

[24] Boellaard R, Oyen WJ, Hoekstra CJ, Hoekstra OS, Visser EP, Willemsen AT, et al The Netherlands protocol for standardisation and quantification of FDG whole body PET studies in multi-centre trials. Eur J Nucl Med Mol Imaging 2008;35:2320-33.

[25] Carvalho S, Leijenaar RTH, Velazquez ER, Oberije C, Parmar C, van Elmpt W, et al. Prognostic value of metabolic metrics extracted from baseline positron emission tomography images in non-small cell lung cancer. Acta Oncol 2013;52:1398-404.

[26] Weistrand O, Svensson S. The ANACONDA algorithm for deformable image registration in radiotherapy. Med Phys 2015;42:40-53.

[27] Lea D, Catcheside D. The mechanism of the induction by radiation chromosome aberrations in Tradescentia. J Genet 1942;44:216-45.

[28] Boellaard R, Delgado-Bolton R, Oyen WJG, Giammarile F, Tatsch K, Eschner W, et al. FDG PET/CT: EANM procedure guidelines for tumour imaging: version 2.0. Eur J Nucl Med Mol Imaging 2015;42:328-54.

[29] Mattoli MV, Massaccesi M, Castelluccia A, Scolozzi V, Mantini G, Calcagni ML. The predictive value of (18)F-FDG PET-CT for assessing the clinical outcomes in locally advanced NSCLC patients after a new induction treatment: low-dose fractionated radiotherapy with concurrent chemotherapy. Radiat Oncol 2017;12:4. 